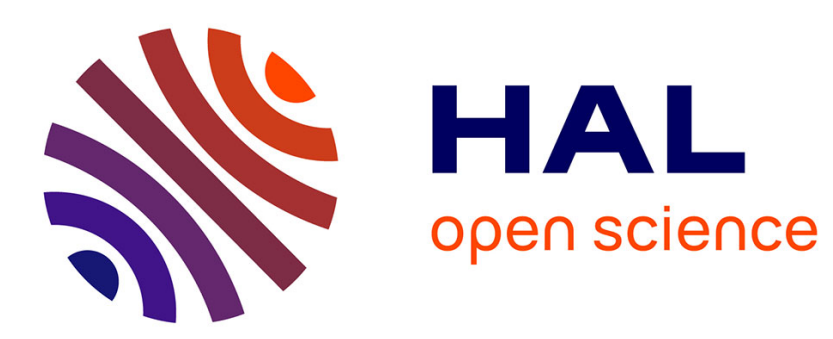

\title{
Measuring similarity among plots including similarity among species: an extension of traditional approaches
}

Carlo Ricotta, Sandrine Pavoine

\section{To cite this version:}

Carlo Ricotta, Sandrine Pavoine. Measuring similarity among plots including similarity among species: an extension of traditional approaches. Journal of Vegetation Science, 2015, 26 (6), pp.1061-1067. $10.1111 /$ jvs.12329 . hal-02552661

\section{HAL Id: hal-02552661 \\ https://hal.science/hal-02552661}

Submitted on 23 Apr 2020

HAL is a multi-disciplinary open access archive for the deposit and dissemination of scientific research documents, whether they are published or not. The documents may come from teaching and research institutions in France or abroad, or from public or private research centers.
L'archive ouverte pluridisciplinaire HAL, est destinée au dépôt et à la diffusion de documents scientifiques de niveau recherche, publiés ou non, émanant des établissements d'enseignement et de recherche français ou étrangers, des laboratoires publics ou privés. 


\section{Measuring similarity among plots including similarity among species:} An extension of traditional approaches

\section{Carlo Ricotta ${ }^{1, *}$ and Sandrine Pavoine ${ }^{2,3}$}

1(corresponding author carlo.ricotta@uniroma1.it) Department of Environmental Biology, University of Rome 'La Sapienza', Rome, Italy; '2(pavoine@mnhn.fr) Département Ecologie et Gestion de la Biodiversité, Muséum National d'Histoire Naturelle, Paris, France; ${ }^{3}$ Department of Zoology, Mathematical Ecology Research Group, University of Oxford, Oxford, UK.

\section{Abstract}

Question: Presence/absence coefficients of (dis)similarity are generally based on various combinations of the matching/mismatching components of the classical $2 \times 2$ contingency table. While a few extensions of the contingency table to species abundance data were proposed, a generalization of such coefficients which includes species resemblances is still lacking.

Methods: In this paper we introduce a new family of plot-to-plot (dis)similarity coefficients, which include species abundances and interspecies resemblances in the calculation of the matching/mismatching components of the contingency table. Within this family, the behavior of five similarity coefficients is examined by graphical comparisons based on simulated data.

Conclusions: This new family of similarity coefficients can equally apply to functional or phylogenetic resemblance among species, thus providing a unified framework for comparing species turnover among plots.

Keywords: Abundance-based coefficients; Contingency table; Interspecies resemblances; Presence/absence coefficients; Species ordinariness.

\section{Introduction}

Ecological studies often rely on similarity coefficients between pairs of species assemblages (or communities, plots, sites, quadrats, etc.) to explore the mechanisms driving community assembly. However, although similarity may appear as a simple and unambiguous notion, when we look for a suitable numerical definition, we find that it is independent of any single way of measurement. Ecologists have developed a rich arsenal of coefficients for expressing the similarity between two species plots and the choice among them is governed by several criteria, the foremost of which is whether composition is described in terms of species presence and absence scores or species abundances (Tamás et al. 2001).

Presence/absence coefficients of similarity are usually formulated based on the matching/mismatching components of the well-known $2 \times 2$ contingency table. These components express the number of species present (a) in both plots, (b) in the first plot but not in the second, (c) in the second plot but not in the first, and (d) the species absent from both plots but found in other plots, such that the sum $S=a+b+c+d$ is the total number of species in the entire collection of plots. Once the values of the matching/mismatching components are determined, any of a large number of similarity and similarity measures for presence/absence data can be calculated, such as the simple matching coefficient (Sokal \& 
Michener 1958) $(a+d) /(a+b+c+d)$ or the Jaccard (1900) index of similarity $(a) /(a+b+c)$. For an exhaustive list of presence/absence similarity measures, together with a throughout discussion of the pros and cons of including double absences (i.e. component d) in the calculation of plot-to-plot similarity coefficients, see Legendre \& Legendre (2012) and Podani (2000).

Unlike presence/absence coefficients, abundance-based measures are usually expressed in terms of the original raw data scores, and this different formalism makes it difficult to generalize presence/absence coefficients to the quantitative case. Among the few attempts to redefine the $2 \times 2$ contingency table to account for species abundances (i.e. Tamás et al. 2001; Chao et al. 2006) we focus here on the approach of Tamás et al. (2001). Let $\mathbf{X}=\left\{\mathrm{x}_{\mathrm{ik}}\right\}$ denote a matrix of $\mathrm{S}$ species $\times \mathrm{N}$ plots with the general element $\mathrm{x}_{\mathrm{ik}}$ representing the abundance of species $\mathrm{i}$ in plot k. According to Tamás et al. (2001), for a pair of plots $\mathrm{k}$ and $\mathrm{h}$, the matching/mismatching components of the contingency table can be defined as follows: using capital letters to represent the abundance-based components, A is the amount of species abundances in which the two plots agree, summed over all species

$$
A=\sum_{i}^{S} \min \left\{x_{i k}, x_{i h}\right\}
$$

$B$ is the sum of species abundances by which plot k exceeds plot h,

$$
B=\sum_{i}^{S}\left(\max \left\{x_{i k}, x_{i h}\right\}-x_{i h}\right)
$$

and $\mathrm{C}$ is the sum of species abundances by which plot $\mathrm{h}$ exceeds plot $\mathrm{k}$

$$
C=\sum_{i}^{S}\left(\max \left\{x_{i k}, x_{i h}\right\}-x_{i k}\right)
$$

$$
\text { To clarify the meaning of } \mathrm{A}, \mathrm{B} \text {, and } \mathrm{C} \text { in a more intuitive manner, imagine species }
$$
abundances are measured using the number of individuals in each plot. In this case, $A$ is the number of individuals of each species shared by both plots $\mathrm{k}$ and $\mathrm{h}$, while $\mathrm{B}$ and $\mathrm{C}$ summarize the number of individuals that are unique to plots $\mathrm{k}$ and $\mathrm{h}$ respectively (Baselga 2013).

Finally, D is defined as the sum of differences between the highest abundance value of each species in the entire collection of plots and the highest abundance value in the pair of plots under scrutiny. First, the maximum abundance value of each species in the entire plot 
collection is determined; according to Tamás et al. (2001) this value represents the "potential abundance' that the species could reach in the study area. The 'mutual absence' of a given species is then obtained as the potential species abundance minus the maximum abundance reached by the species in plots $\mathrm{k}$ and $\mathrm{h}$. The summation of the mutual absences over all species in the $\mathrm{S} \times \mathrm{N}$ matrix of species' abundances in plots gives the component $\mathrm{D}$ :

$$
D=\sum_{i}^{S}\left(\max _{k}\left\{x_{i k}\right\}-\max \left\{x_{i k}, x_{i h}\right\}\right)
$$

\section{Although mutual absences are generally not present in the formulation of abundance-based} similarity measures, the component D is included in Tamás et al. (2001) to allow full correspondence between the matching/mismatching components of the $2 \times 2$ contingency table for presence/absence data and its abundance-based counterpart.

While the work of Tamás et al. (2001) allowed to generalize the matching/mismatching components of the usual $2 \times 2$ contingency table to species abundances, it has been recently recognized that similarity measures should also incorporate information about the degree of ecological differences between species (i.e. Nipperess et al. 2010; Chiu et al. 2014; Villéger et al. 2013; Pavoine \& Ricotta 2014; Ricotta et al. 2015). Obviously, the notion of interspecies differences is multifaceted, including functioning (Diáz \& Cabido 2001), morphology (Roy \& Foote 1997), or evolutionary history (Clarke et al. 2006). In a phylogenetic context, Ferrier et al. (2007) proposed a method for calculating the (dis)similarity between the species presences and absences in two plots based on the amount of total branch length unique to those plots. Accordingly, two well-known coefficients of phylogenetic dissimilarity, the UniFrac measure (Lozupone \& Knight 2005), and the PhyloSor measure (Bryant et al. 2008) have since been special cases of the family of measures of Ferrier et al. (2007). By adopting the approach of Tamás et al. (2001), Nipperess et al. (2010) further extended this method to incorporate species abundance data into phylogenetic dissimilarity measures. Irrespective of the way interspecies resemblances are measured, the main objective of this study is to generalize the work of Tamás et al. (2001) to introduce a new family of plot-to-plot similarity coefficients, which include interspecies resemblances in the calculation of the matching/mismatching components of a $2 \times 2$ contingency table (hereafter ISR-based measures, where ISR stands for 'InterSpecies Resemblances'). 
Regardless of their nature, interspecies differences are usually represented by a square $S \times S$ symmetric matrix $\mathbf{D}=\left\{\mathrm{d}_{\mathrm{ij}}\right\}$ of (functional, morphological or phylogenetic) dissimilarities between a pair of species $\mathrm{i}$ and $\mathrm{j}$ with $\mathrm{d}_{\mathrm{ij}}=\mathrm{d}_{\mathrm{ji}}$ and $\mathrm{d}_{\mathrm{ii}}=0$. If the dissimilarity coefficient $\mathrm{d}$ is comprised in the range $[0,1]$, it is possible to define a corresponding similarity coefficient $\mathrm{s}$ as the complement of $\mathrm{d}: \mathrm{s}=1-\mathrm{d}$. For any dissimilarity measure with an upper bound $d_{\max }>$

1131 , division by $d_{\max }$ produces a normalized dissimilarity measure in the range [0, 1]. For 114 dissimilarity measures that do not possess an upper bound, such as phylogenetic distances, it 115 is still possible to locally normalize the values $d_{i j}$ in the range $[0,1]$ by dividing each term by 116 the maximum value found in the data set.

117 Like in Leinster \& Cobbold (2012), based on species abundances and similarities, we can 118 define the (absolute) abundance of species similar to $\mathrm{i}$ in plot $\mathrm{k}\left(\mathrm{Z}_{\mathrm{ik}}\right)$ as:

$$
Z_{i k}=\sum_{j}^{S(k h)} x_{j k} s_{i j}
$$

where $S(k h)$ is the number of species in the pool of plots $k$ and $h$ (i.e. the species for which $\min \left\{x_{i k}, x_{i h}\right\}>0$ ). Neglecting within-species differences (i.e. setting $\mathrm{s}_{\mathrm{ii}}=1$ by definition) we always have that the relative abundance of all species similar to $i$ (including $i$ itself) cannot be lower than the abundance of species i $\left(x_{i k} \leq Z_{i k} \leq \sum_{i} x_{i k}\right.$, i.e. the total species abundance in plot $\mathrm{k}) . \mathrm{Z}_{\mathrm{ik}}$ is large if most species in the assemblage are similar to i. On the other hand, if species $\mathrm{i}$ is very dissimilar from the other species $\mathrm{Z}_{\mathrm{ik}} \approx \mathrm{X}_{\mathrm{ik}}$. The quantity $\mathrm{Z}_{\mathrm{ik}}$ thus measures the ordinariness of species i. Starting from the species ordinariness $Z_{i k}$, we can now expand the method of Tamás et al. (2001) to calculate a generalized version of the matching/mismatching components of the $2 \times 2$ contingency table including information on interspecies resemblances.

131 This is obtained simply by substituting the quantities $Z_{i k}$ for $x_{i k}$ in Eqs. (1-3):

$$
A=\sum_{i}^{S(k h)} \min \left\{Z_{i k}, Z_{i h}\right\}
$$

$135 \quad B=\sum_{i}^{S(k h)}\left(\max \left\{Z_{i k}, Z_{i h}\right\}-Z_{i h}\right)$ 
$137 \quad C=\sum_{i}^{S(k h)}\left(\max \left\{Z_{i k}, Z_{i h}\right\}-Z_{i k}\right)$

138

139

140

141

142

143

144

145

146

147

148

150

151

152

153

154

155

156

157

158

159

160

161

162

163

164

165

166

167

Like for Eq. (5), the sum in Eqs. (6-8) is over all species $S(k h)$. In other words, it is over all species that are actually present in at least one of the two plots $\mathrm{h}$ and $\mathrm{k}$, without reference to the species absent from both plots but found in other plots of the $\mathrm{S} \times \mathrm{N}$ matrix of species' abundances in plots (see Leinster \& Cobbold 2012; Ricotta et al. 2015).

According to this restrictive definition of the A, B and C components, a formal definition of the D component makes only little sense. This is because the calculation of the A, B and C components in Eqs. (6-8) is based on a plot-level species pool, whereas the calculation of the term $\max _{k}\left\{Z_{i k}\right\}$ of the D component (see Eq. 4) usually refers to the matrix-level species pool. Nonetheless, for sake of completeness and in analogy with Tamás et al. (2001), a less restrictive definition of the $\mathrm{A}, \mathrm{B}$ and $\mathrm{C}$ components which takes into account all species in the $\mathrm{S} \times \mathrm{N}$ matrix, together with the corresponding D component, is shown in Appendix S1. In Appendix S1 an alternative definition of the new A, B, C and D components, which is based on species relative abundances instead of absolute abundances is also shown.

Starting from this generalized definition of the $2 \times 2$ contingency table, virtually all presence/absence similarity coefficients can be generalized to include ecological differences among species, thus providing a new family of measures. Note that for zero interspecies similarities (i.e. $s_{i j}=0$ for $i \neq j$ ) Eqs. (6-8) recover the A, B and C components of Tamás et al. (2001), while for zero interspecies similarities and unit abundances Eqs. (6-8) recover the a, b and $\mathrm{c}$ matching/mismatching components of the traditional $2 \times 2$ contingency table.

\section{Case Study}

The relationship between selected similarity coefficients calculated from the generalized contingency table and their traditional presence/absence counterparts were investigated by graphical evaluation of artificial data representing an ideal ecological gradient (Gower \& Legendre 1986; Tamás et al. 2001; Podani \& Miklós 2002). The basis for the comparative evaluation is the $\mathrm{S} \times \mathrm{N}$ matrix in Table 1 in which 9 Plots (P1-P9) are characterized in terms of 15 quantitative variables (the abundances of species S1-S15). The matrix was constructed such that all species have a unimodal response of varying length and intensity (abundance) to a hypothetical one-dimensional gradient, such that the species optima are located at different 
positions along the gradient. In some cases this unimodal response is truncated at one extreme of the gradient (see e.g. Species S1 or S14 in Table 1). The comparison of plot P1 with itself and with the remaining plots provides nine similarity values. We used profile diagrams to show the effect of species turnover upon the similarities.

In this paper we compared the presence/absence version of five traditional similarity

173 coefficients, the Jaccard, Sokal-Sneath, Sørensen, Ochiai and Kulczynski indices (formulas in Table 2) with their abundance-based analogues (calculated with Eqs. 1-3) and with three different ISR-based analogues (all calculated with Eqs. 6-8 but with different interspecies similarity matrices).

For the calculation of the presence/absence coefficients, the abundance data of Table 1 were converted to binary presence and absence data. For the calculation of the ISR-based coefficients, we used three different interspecies similarity matrices (M1-M3; see Appendix S2). In matrix M1, interspecies similarities reflect the species ecological differences along the artificial gradient of Table 1. To this end, interspecies similarities were set roughly proportional to the distance between the locations of the species optima and to the difference between their optimal abundances. For example, in Table 1, the optimum of species S9 is located in plot P4 while the optimum of species S12 is located in plot P8. Accordingly, the distance between their optima along the ideal gradient of Table 1 is $O d=4$. Likewise, the difference in their optimal abundances is $O a=4-2=2$. Based on these quantities, the interspecies similarity $\mathrm{s}_{\mathrm{ij}}$ between species $\mathrm{S} 9$ and $\mathrm{S} 12$ was calculated as: $s_{i j}=1-(O d+O a) / 10$. Therefore, for the specific case of $\mathrm{S} 9$ and $\mathrm{S} 12$, $s_{i j}=1-(4+2) / 10=0.4$. Note that if $O d+O a>10, s_{i j}=0$ by definition, such that the species $\mathrm{i}$ and $\mathrm{j}$ are considered maximally dissimilar from each other. Note also that, according to this way of calculating interspecies distances, species S1 and S2 in Table 1 are maximally similar to each other, meaning that for this pair of species $s_{i j}=1$. Finally, in matrix M2 interspecies similarities were generated assigning to each pair of species a value $0 \leq s_{i j} \leq 1$ drawn at random from an even distribution, while in $\mathbf{M 3}$ all interspecies similarities were uniformly set to $s_{i j}=0.33$.

For each similarity index in Table 2 we calculated i) its presence/absence version, ii) its abundance-based version according to Eqs. (1-3) and iii) three ISR-based versions (using the interspecies similarity matrices M1-M3 in Appendix S2). All ISR-based indices were calculated according to the distinct definitions of the matching/mismatching components 
proposed in this paper (i.e. Eqs. 6-8 and Eqs. A1-A3, A5-A7, and A10-A12 in Appendix S1). All calculations were performed with the new R script available in Appendix S3.

\section{Results}

For the artificial ecological gradient in Table 1, the analyses performed with the various definitions of the matching/mismatching components proposed in this paper yielded qualitatively similar results (see Appendix S4). Therefore, we report here only results from the ISR-based indices calculated with Eqs. (6-8). Figure 1 illustrates the IRS-based version of the indices in Table 2 calculated with Matrix M1. In Figure 1, the profiles of all similarity coefficients show essentially the same pattern, meaning that, although all coefficients summarize plot-to-plot similarity from different viewpoints, they generally show a consistent behavior in response to the artificial ecological gradient in Table 1. The profiles of the Sørensen, Ochiai and Kulczynski coefficients have very similar shapes. This is not surprising, as for identical $b$ and $c$ components all three indices reduce to the same expression $a /(a+b)$.

Figure 2(a-c), illustrates the graphical comparison among the presence/absence versions of the Jaccard, Sokal-Sneath and Ochiai coefficients with their abundance-based and ISR-based analogues. Due to their similarity with the Ochiai index, the Sørensen and Kulczynski coefficients are not shown here but see Appendix S4. In all cases, the curves of the presence/absence indices and of the abundance-based indices show a non-linear decreasing pattern in response to the artificial gradient of Table 1. The same decreasing behavior, although with a different curve shape, is shown by the ISR-based measures calculated with matrix M1. That is, if interspecies similarities $s_{\mathrm{ij}}$ reflect to some degree the species sorting along the gradient of Table 1, the ISR-based index is able to rank the plot-to-plot similarities correctly. However, due to the non-zero values of most interspecies similarities in matrix M1, the plot-to-plot similarity values of the ISR-based indices are systematically larger than the corresponding presence/absence and abundance-based indices.

To the contrary, the ISR-based indices calculated with matrices M2 and M3 rank the plotto-plot similarity values differently with a number of distinct local maxima and minima that are absent from the other index profiles. Hence, unlike for presence/absence and abundancebased indices, the similarity values of the ISR-based coefficients depend on the complex interplay of species abundances and interspecies similarities. Note here that the similar shapes of the ISR-based index profiles calculated with matrix M2 and M3 mean that on average a random distribution of interspecies similarities (matrix M2) produces the same results as the uniform distribution of $\mathrm{s}_{\mathrm{ij}}$ values of matrix M3. 


\section{Discussion}

235 In this paper we developed a methodological framework for measuring the similarity among plots including the similarity among species within plots. The proposed framework is very flexible in the calculation of interspecies similarities, which can be obtained in a variety of ways from phylogenetic or functional data sets containing a combination of scale types (i.e. quantitative, ordinal, nominal and binary variables). This flexibility is particularly desirable, as it is now well-established that a single measure is unable to summarize all aspects of plotto-plot similarity. Juhasz-Nágy (1993; see also Podani et al. 2005) first imagined the measurement of an ecological variable, such as a similarity coefficient, as an iterative process involving successive levels of increasing abstraction (i.e. from presence/absence-measures to abundance-based measures, and to ISR-based measures). At each step of this 'complex, plural and dynamic approach' (sensu Juhasz-Nagy), the view angle is restricted to zoom on a specific aspect of the given problem, increasing at the same time the number of possible viewpoints.

In this paper, the extension of presence/absence indices to measures including interspecies resemblances, offers a number of different options that are shown in the main text (Eqs. 6-8) and in Appendix S1, such that practitioners should determine which definition of the generalized contingency table is more adequate within the context of their analyses. Nonetheless, the suggested index generalizations were not proposed as a mere addition to the dozens of available similarity measures. Rather, they enable plot-to-plot comparisons at several levels of increasing biotic complexity with the exclusion of confounding effects that may be due to differences in the way the indices are computed, and that may mask the ecological processes under scrutiny (Tamás et al. 2001).

Comparing the new indices, Figure 1 shows that, while the new ISR-based measures tend to rank plots in the same order, nonetheless the index values can be highly variable depending on how the three $\mathrm{A}, \mathrm{B}$ and $\mathrm{C}$ matching/mismatching components are combined. Therefore, to compare two species assemblages, the same index needs to be used. This is a well-known property of the standard indices that use a, b, c matching/mismatching components. Indeed once an index has been chosen (for example the Jaccard index), care should be taken in interpreting and communicating the results of the index. While most similarity indices proposed for combining the $\mathrm{a}, \mathrm{b}, \mathrm{c}$ matching/mismatching components vary between the same limits (0 and 1), they usually do not provide comparable similarity values for the same pair of plots. This renders the automatic conversion of the index values to a qualitative scale, such as very low, low, moderate, high, and very high (dis)similarity based on a 0.2 interval scale 
impossible, thus making cross-study comparisons with different indices highly misleading

269 (Bloom 1981). This well-known property of standard indices also applies to the new ISRbased measures. On the other hand, as the different indices have slightly different properties, they summarize plot-to-plot similarity from different viewpoints (Pavoine \& Ricotta 2014).

Depending on interspecies resemblances and unlike conventional presence/absence or 273 abundance-based indices, ISR-based indices usually provide non-zero similarity even for a 274 pair of plots with no species in common, as different species may share some degree of 275 similarity due to common phylogenetic ancestry and/or common functional characteristics. 276 This is a very useful property if one wishes to calculate, e.g., the functional similarity among 277 plots under similar environmental conditions in different regions of the world. In addition, it 278 has been recently shown that dissimilarities among communities result from two different 279 processes: species turnover (or replacement) and species gain and loss, also called richness 280 difference or nestedness (Lennon et al. 2001; Baselga 2010). While the debate concerning the 281 best ways of additively partitioning dissimilarity coefficients is ongoing (e.g. Carvalho et al. 282 2013; Legendre 2014), Podani \& Schmera (2011) and Carvalho et al. (2012) independently 283 proposed to decompose the Jaccard dissimilarity $1-(a) /(a+b+c)=(b+c) /(a+b+c)$ into 284 two additive portions: species replacement $2 \min \{b, c\} /(a+b+c)$ and richness difference $285|b-c| /(a+b+c)$. Podani et al. (2013) next proposed an extension of the Jaccard dissimilarity 286 decomposition to abundance data. Based on the generalization of the matching/mismatching 287 components of the contingency table proposed in this paper, the additive dissimilarity 288 decomposition can be further extended to ISR-based dissimilarities using a unified algebraic 289 framework.

290 To conclude, we hope the proposed indices will help shedding light on new aspects of 291 ecological data that would remain undetected using standard similarity measures that do not include (phylogenetic and functional) information on the species. 


\section{References}

Baselga, A. 2010. Partitioning the turnover and nestedness components of beta diversity. Global Ecology and Biogeography 19: 134-143.

Bloom, S.A. 1981. Similarity indices in community studies: potential pitfalls. Marine Ecology Progress Series 5: 125-128.

Bryant, J.A., Lamanna, C., Morlon, H., Kerkhoff, A.J., Enquist, B.J. \& Green, J.L. 2008. Microbes on mountainsides: contrasting elevational patterns of bacterial and plant diversity. Proceedings of the National Academy of Sciences USA 105: 11505-11511.

Carvalho, J.C., Cardoso, P. \& Gomes, P. 2012. Determining the relative roles of species replacement and species richness differences in generating beta-diversity patterns. Global Ecology and Biogeography 21: 760-771.

Carvalho, J.C., Cardoso, P., Borges, P.A.V., Schmera, D. \& Podani, J. 2013. Measuring fractions of beta diversity and their relationships to nestedness: a theoretical and empirical comparison of novel approaches. Oikos 122: 825-834.

Chao, A., Chazdon, R.L., Colwell, R.K. \& Shen, T.J. 2006. Abundance-based similarity indices and their estimation when there are unseen species in samples. Biometrics 62: 361371.

Chiu, C.-H., Jost, L. \& Chao, A. 2014. Phylogenetic beta diversity, similarity, and differentiation measures based on Hill numbers. Ecological Monographs 84: 21-44.

Clarke, K.R., Somerfield, P.J. \& Chapman, M. 2006. On resemblance measures for ecological studies, including taxonomic dissimilarities and a zero-adjusted Bray-Curtis coefficient for denuded assemblages. Journal of Experimental Marine Biology and Ecology 330: 55-80.

Diáz, S. \& Cabido, M. 2001. Vive la différence: plant functional diversity matters to ecosystem processes. Trends in Ecology and Evolution 16: 646-655.

Ferrier, S., Manion, G., Elith, J. \& Richardson, K. 2007. Using generalized dissimilarity modelling to analyse and predict patterns of beta diversity in regional biodiversity assessment. Diversity and Distributions 13: 252-264.

Gower, J.C. \& Legendre, P. 1986. Metric and Euclidean properties of dissimilarity coefficients. Journal of Classification 3: 5-48.

Jaccard, P. 1900. Contribution au problème de l'immigration post-glaciaire de la flore alpine. Bulletin de la Société Vaudoise des Sciences Naturelles 36: 87-130.

Juhász-Nagy, P. 1993. Notes on compositional diversity. Hydrobiologia 249: 173-182.

Legendre, P. \& Legendre, L. 2012. Numerical ecology, Elsevier Science BV, Amsterdam.

Legendre, P. 2014. Interpreting the replacement and richness difference components of beta diversity. Global Ecology and Biogeography doi: 10.1111/geb.12207.

Leinster, T. \& Cobbold, C.A. 2012. Measuring diversity: the importance of species similarity. Ecology 93: 477-489.

Lennon, J.J., Koleff, P., Greenwood, J.J.D. \& Gaston, K.J. 2001. The geographical structure of British bird distributions: diversity, spatial turnover and scale. Journal of Animal Ecology 70: 966-979.

Lozupone, C.A. \& Knight, R. 2005. UniFrac: a new phylogenetic method for comparing microbial communities. Applied and Environmental Microbiology 71: 8228-8235.

Nipperess, D.A., Faith, D.P. \& Barton, K. 2010. Resemblance in phylogenetic diversity among ecological assemblages. Journal of Vegetation Science 21: 809-820.

Ochiai, A. 1957. Zoogeographic studies on the soleoid fishes found in Japan and its neighbouring regions. Bulletin of the Japanese Society of Scientific Fisheries 22: 526-530.

Pavoine, S. \& Ricotta, C. 2014. Functional and phylogenetic similarity among communities. Methods in Ecology and Evolution doi: 10.1111/2041-210X.12193.

Podani, J. \& Schmera, D. 2011. A new conceptual and methodological framework for exploring and explaining pattern in presence-absence data. Oikos 120: 1625-1638. 
Podani, J. 2000. Introduction to the Exploration of Multivariate Biological Data. Backhuys, Leiden.

Podani, J. \& Miklós, I., 2002. Resemblance coefficients and the horseshoe effect in principal coordinates analysis. Ecology 83: 3331-3343.

Podani, J., Csontos, P., Tamás, J. \& Miklós, I. 2005. A new multivariate approach to studying temporal changes of vegetation. Plant Ecology 181: 1-16.

Podani, J., Ricotta, C. \& Schmera, D. 2013. A general framework for analyzing beta diversity, nestedness and related community-level phenomena based on abundance data. Ecological Complexity 15: 52-61.

Ricotta, C., Bacaro, G. \& Pavoine, S. 2015. A cautionary note on some phylogenetic dissimilarity measures. Journal of Plant Ecology 8: 12-16.

Roy, K. \& Foote, M. 1997. Morphological approaches to measuring biodiversity. Trends in Ecology and Evolution 12: 277-281.

Sokal, R. \& Michener, C. 1958. A statistical method for evaluating systematic relationships. University of Kansas Scientific Bulletin 38: 1409-1438.

Sokal, R.R. \& Sneath, P.H.A. 1963. Principles of Numerical Taxonomy. Freeman, San Francisco.

Sørensen, T. 1948. A method of establishing groups of equal amplitude in plant sociology based on similarity of species content. Kongelige Danske Videnskabernes Selskabs Biologiske Skrifter 5: 1-34.

Tamás, J., Podani, J. \& Csontos, P. 2001. An extension of presence/absence coefficients to abundance data: a new look at absence. Journal of Vegetation Science 12: 401-410.

Villéger, S., Grenouillet, G. \& Brosse, S. 2013. Decomposing functional $\beta$-diversity reveals that low functional $\beta$-diversity is driven by low functional turnover in European fish 
370 Additional Supporting Information may be found in the online version of this article.

\section{Appendix S1}

Definition of some alternative versions of the ISR-based matching/mismatching components of the generalized $2 \times 2$ contingency table.

\section{Appendix S2}

Interspecies similarity matrices M1-M3 used for the calculation of the ISR-based versions of indices in Table 2 (their associated dissimilarity matrices, i.e. 1-M1, 1-M2 and 1-M3, respectively, are given as text files in Appendix S6 to S8).

\section{Appendix S3}

$\mathrm{R}$ script for the calculation of the similarity indices.

\section{Appendix $\mathbf{S 4}$}

Manual for using the R script and examples. The examples are based on Table 1 in the main text (see Appendix S5) and on the dissimilarity matrices 1-M1, 1-M2 and 1-M3 (see Appendix S6 to S8).

\section{Appendix $\mathbf{5 5}$}

Table 1 in text format.

\section{Appendix $\mathbf{5 6}$}

Dissimilarity matrix 1-M1 in text format.

\section{Appendix $\mathbf{5 7}$}

Dissimilarity matrix 1-M2 in text format.

\section{Appendix $\mathbf{S 8}$}

400 
Table 1. Artificial data matrix for the graphical comparison of the indices in Table 2. The data represent an ideal ecological gradient with simplified unimodal species responses of varying length and abundance. The matrix was generated randomly and then rearranged according to a sequential location of species optima from P1 to P9. Non-zero species abundances are shown in grey. The locations of all species optima are shown in bold.

\begin{tabular}{|c|ccccccccc|}
\cline { 2 - 9 } \multicolumn{1}{c|}{} & \multicolumn{10}{c|}{ Plots } \\
\hline Species & P1 & P2 & P3 & P4 & P5 & P6 & P7 & P8 & P9 \\
\hline S1 & $\mathbf{2}$ & 1 & 0 & 0 & 0 & 0 & 0 & 0 & 0 \\
S2 & $\mathbf{2}$ & 1 & 0 & 0 & 0 & 0 & 0 & 0 & 0 \\
S3 & $\mathbf{3}$ & 2 & 1 & 0 & 0 & 0 & 0 & 0 & 0 \\
S4 & $\mathbf{4}$ & 3 & 2 & 1 & 0 & 0 & 0 & 0 & 0 \\
S5 & 0 & $\mathbf{1}$ & 0 & 0 & 0 & 0 & 0 & 0 & 0 \\
S6 & 0 & 0 & $\mathbf{1}$ & 0 & 0 & 0 & 0 & 0 & 0 \\
S7 & 2 & 3 & $\mathbf{4}$ & 3 & 2 & 1 & 0 & 0 & 0 \\
S8 & 0 & 1 & 2 & $\mathbf{3}$ & 2 & 1 & 0 & 0 & 0 \\
S9 & 1 & 2 & 3 & $\mathbf{4}$ & 3 & 2 & 1 & 0 & 0 \\
S10 & 0 & 0 & 0 & 1 & $\mathbf{2}$ & 1 & 0 & 0 & 0 \\
S11 & 0 & 0 & 0 & 0 & 0 & $\mathbf{1}$ & 0 & 0 & 0 \\
S12 & 0 & 0 & 0 & 0 & 0 & 0 & 1 & $\mathbf{2}$ & 1 \\
S13 & 0 & 0 & 0 & 0 & 1 & 2 & 3 & 4 & $\mathbf{5}$ \\
S14 & 0 & 0 & 0 & 0 & 0 & 0 & 1 & 2 & $\mathbf{3}$ \\
S15 & 0 & 0 & 0 & 0 & 0 & 0 & 0 & 1 & $\mathbf{2}$ \\
\hline
\end{tabular}

Table 2. Traditional similarity coefficients for presence/absence data used in this study. Only coefficients ignoring the value of the d component were considered.

\begin{tabular}{|c|c|c|}
\hline Index Name & Formula & Source \\
\hline Jaccard & $\frac{a}{a+b+c}$ & Jaccard (1900) \\
\hline Sørensen & $\frac{2 a}{2 a+b+c}$ & Sørensen (1948) \\
\hline Ochiai & $\frac{a}{\sqrt{(a+b)(a+c)}}$ & Ochiai (1957) \\
\hline Kulczynski & $\frac{1}{2}\left[\frac{a}{(a+b)}+\frac{a}{(a+c)}\right]$ & Sokal and Sneath (1963) \\
\hline Sokal-Sneath & $\frac{a}{a+2 b+2 c}$ & Sokal and Sneath (1963) \\
\hline
\end{tabular}

